\title{
Splenosis mimicking gastric obstructive tumor. Diagnostic workup.
}

Vaia-Aliki Rompou ${ }^{1}$, Dimitrios Korkolis ${ }^{1}$, Evelina Skafida ${ }^{1}$, Dimitrios Tsamis ${ }^{2}$, and Aris Plastiras $^{1}$

${ }^{1}$ Agios Savvas General Cancer and Oncology Hospital of Athens

${ }^{2}$ Athens Medical Centre

May 31, 2021

\begin{abstract}
Splenosis is a condition that occurs after splenic rupture. A 29 year-old male patient with a history of splenectomy, was admitted due to multiple vomiting episodes. The diagnostic workup was unable to differentiate between gastric GIST and splenosis. Laparoscopic surgical resection was performed leading to the diagnosis of splenosis.
\end{abstract}

\section{Hosted file}

Splenosis mimicking gastric obstructive tumor. Diagnostic workup. .docx available at https: //authorea.com/users/417068/articles/524305-splenosis-mimicking-gastric-obstructivetumor-diagnostic-workup 